University of Nebraska - Lincoln

DigitalCommons@University of Nebraska - Lincoln

Faculty Publications from the Harold W. Manter Laboratory of Parasitology

2000

\title{
MOLECULAR SYSTEMATICS OF MESOCESTOIDES SPP. (CESTODA: MESOCESTOIDIDAE) FROM DOMESTIC DOGS (CANIS FAMILIARIS) AND COYOTES (CANIS LATRANS)
}

\author{
Paul R. Crosbie \\ University of California - Davis \\ Steven A. Nadler \\ University of California - Davis, sanadler@ucdavis.edu \\ Edward G. Platzer \\ University of California - Riverside \\ Cynthia Kerner \\ Muséum d'Histoire Naturelle \\ J. Mariaux \\ Muséum d'Histoire Naturelle \\ See next page for additional authors \\ Follow this and additional works at: https://digitalcommons.unl.edu/parasitologyfacpubs \\ Part of the Parasitology Commons
}

Crosbie, Paul R.; Nadler, Steven A.; Platzer, Edward G.; Kerner, Cynthia; Mariaux, J.; and Boyce, Walter M., "MOLECULAR SYSTEMATICS OF MESOCESTOIDES SPP. (CESTODA: MESOCESTOIDIDAE) FROM DOMESTIC DOGS (CANIS FAMILIARIS) AND COYOTES (CANIS LATRANS)" (2000). Faculty Publications from the Harold W. Manter Laboratory of Parasitology. 707.

https://digitalcommons.unl.edu/parasitologyfacpubs/707

This Article is brought to you for free and open access by the Parasitology, Harold W. Manter Laboratory of at DigitalCommons@University of Nebraska - Lincoln. It has been accepted for inclusion in Faculty Publications from the Harold W. Manter Laboratory of Parasitology by an authorized administrator of DigitalCommons@University of Nebraska - Lincoln. 


\section{Authors}

Paul R. Crosbie, Steven A. Nadler, Edward G. Platzer, Cynthia Kerner, J. Mariaux, and Walter M. Boyce 


\title{
MOLECULAR SYSTEMATICS OF MESOCESTOIDES SPP. (CESTODA: MESOCESTOIDIDAE) FROM DOMESTIC DOGS (CANIS FAMILIARIS) AND COYOTES (CANIS LATRANS)
}

\author{
Paul R. Crosbie, Steven A. Nadler , Edward G. Platzer†, Cynthia Kerner, J. Mariaux $\ddagger$, and Walter M. Boyce $\S$ \\ Department of Veterinary Pathology, Microbiology and Immunology, University of California, Davis, One Shields Avenue, Davis, \\ California 95616-8739
}

\begin{abstract}
The genus Mesocestoides Vaillant, 1863 includes tapeworms of uncertain phylogenetic affinities and with poorly defined life histories. We previously documented 11 cases of peritoneal cestodiasis in dogs (Canis familiaris L.) in western North America caused by metacestodes of Mesocestoides spp. In the current study, DNA sequences were obtained from metacestodes collected from these $\operatorname{dogs}(\mathrm{n}=10)$, as well as proglottids from dogs $(\mathrm{n}=3)$ and coyotes (Canis latrans Say, 1823 [ $\mathrm{n}=2])$, and tetrathyridia representing laboratory isolates of $M . \operatorname{corti}(\mathrm{n}=3)$, and these data were analyzed phylogenetically. Two nuclear genetic markers, 18S ribosomal DNA and the second internal-transcribed spacer (ITS 2), were sequenced. Phylogenetic analysis of the 18S rDNA data recovered a monophyletic group composed of all samples of Mesocestoides spp., distinct from closely related outgroup taxa (Amurotaenia Akhmerov, 1941 and Tetrabothrius Rudolphi, 1819). Initial analysis of the ITS 2 data resolved 3 clades within Mesocestoides. Two proglottids from dogs formed a basal clade, a second clade was represented by tetrathyridial isolates, and a third clade included all other samples. Interpretation of these data from an apomorphy-based perspective identified 6 evolutionary lineages. We also assessed whether metacestodes from dogs $(n=4)$ are capable of asexual proliferation in laboratory mice. One tetrathyridial and 2 acephalic isolates from dogs proliferated asexually. Further investigation is warranted to determine which of the lineages represent distinct species and to determine the life history strategies of Mesocestoides spp.
\end{abstract}

Mesocestoides Vaillant, 1863 spp. have long been recognized as possessing several characteristics that make them distinct from all other cyclophyllidean tapeworms. For example, the median ventral position of the genital atrium and bipartite vitelline gland are unique within the Cyclophyllidea. Another singular feature may be the inferred requirement for 3 hosts in the life cycle (Rausch, 1994). The asexual reproduction of tetrathyridial metacestodes by longitudinal fission originally described by Specht and Voge (1965) is also unique but does not appear to be a universal characteristic of Mesocestoides spp. To date, similar proliferation of tetrathyridia representing other isolates of Mesocestoides spp. has not been unequivocally described and may be rare (Conn, 1990). The tetrathyridia that Specht and Voge (1965) isolated from the fence lizard Sceloporus occidentalis biseriatus (Hallowell, 1854) were identified as tetrathyridia of Mesocestoides corti Hoeppli, 1925, which was originally described from adult worms taken from the house mouse Mus musculus L. (Hoeppli, 1925). Samples of these tetrathyridia were passed from laboratory to laboratory throughout the world and have been referred to as $M$. corti. However, the identification of these tetrathyridia has been questioned, and Mesocestoides vogae $\mathrm{n}$. sp. has been suggested as a binomen (Etges, 1991).

The phylogeny and systematics of the Mesocestoididae are also unresolved. Identification of species of Mesocestoides based on morphological characters is extremely difficult due to high levels of variability. The exclusion of the family from the Cyclophyllidea was thought desirable by Wardle and McLeod (1952), although they concluded that at that time inclusion was unavoidable. Later, ordinal rank was suggested, without any

Received 20 May 1999; revised 16 August 1999; accepted 16 August 1999.

* Department of Nematology, University of California, Davis, One Shields Avenue, Davis, California 95616-8668.

$\dagger$ Department of Nematology, University of California, Riverside, California 92521-0415.

† Muséum d'Histoire Naturelle, CP 6434, 1211 Genève 6, Switzerland. $\S$ Corresponding author. additional evidence (Wardle et al., 1974). Brooks et al. (1991) also recognized Mesocestoides as a problematic group and suggested their exclusion from the Cyclophyllidea. Based on comparative morphology, a basal position for the mesocestoidids (along with the Nematotaeniidae) among putatively cyclophyllidean families has been recommended (Hoberg, Jones, and Bray, 1999). Recent phylogenetic analyses based on partial sequences of nuclear $18 \mathrm{~S}$ ribosomal DNA (rDNA) suggested that the Mesocestoididae be excluded from the Cyclophyllidea because Mesocestoides was basal to all other cyclophyllideans and Tetrabothrius Rudolphi, 1819 (Mariaux, 1998). Additionally, spermatozoon ultrastructure and the pattern of spermiogenesis in Mesocestoides literratus (Goeze, 1782) Raillet, 1893 are different from other cyclophyllideans studied (Miquel et al., 1999).

The impetus for our investigations was the occurrence of numerous cases of peritoneal cestodiasis in domestic dogs $(\mathrm{Ca}$ nis familiaris L.) in western North America caused by metacestodes of Mesocestoides spp. (Crosbie, Boyce, Platzer et al., 1998). With a single exception, the metacestodes collected from these dogs were not tetrathyridia, and the only identifiable cestode structures were calcareous corpuscles. The acephalic metacestodes in these cases were identified as Mesocestoides spp. on the basis of genus-specific patterns for restriction fragment length polymorphism (RFLP) of the second internal transcribed spacer region (ITS 2) of nuclear rDNA (Crosbie, Boyce, Platzer et al., 1998). Although this polymerase chain reaction (PCR)based test was diagnostically sensitive, it did not provide information about the potential genetic diversity of these metacestodes.

To investigate the phylogenetic diversity of Mesocestoides collected from canids, we sequenced portions of 2 genes from the larval specimens of Mesocestoides spp. that we investigated previously (Crosbie, Boyce, Platzer et al., 1998) and from several additional samples (both metacestodes and proglottids). We then used these nucleotide sequences to develop and test phylogenetic hypotheses. To evaluate if these samples were more closely related to each other than to other tapeworm genera, we sequenced a portion of nuclear $18 \mathrm{~S}$ rDNA, as was done pre- 
viously to estimate familial relationships in the Eucestoda (Mariaux, 1998). Sequences from this gene have proved informative for higher level phylogenetic inference and have provided some resolution in a broad range of platyhelminth taxa, including cestodes, digeneans, and turbellarians (Baverstock et al., 1991; Barker et al., 1993; Blair, 1993; Blair and Barker, 1993; Katayama et al., 1993, 1996; Lumb et al., 1993; Rohde et al., 1993, 1994; Carranza et al., 1998). Additionally, sequences from this genetic marker have been used to assess the phylogenetic relationships of Platyhelminthes to other multicellular eukaryotes (Carranza et al., 1997; Campos et al., 1998).

To assess the subgeneric phylogenetic diversity of larval and adult cestodes recovered from canids, we sequenced the 650base pair (bp) ITS 2 region amplified for our RFLP study (Crosbie, Boyce, and Platzer et al., 1998). Previous studies of platyhelminth taxa have shown ITS 2 data to be useful in characterization of taeniid cestode species (Gasser and Chilton, 1993) and highly informative for estimation of subfamilial phylogenies, including the resolution of intraspecific relationships within digeneans (Adlard et al., 1993).

To evaluate whether asexual reproduction of metacestodes can occur in isolates other than $M$. corti, we inoculated groups of laboratory mice with metacestodes recovered from 4 different dogs. Additionally, for comparative and control purposes we also inoculated groups of mice with laboratory isolates of M. corti and Taenia crassiceps (Zeder, 1800) Rudolphi, 1810, both known to be asexually proliferative in mice.

In summary, the general objectives for this study were to: (1) determine whether all specimens of Mesocestoides spp. from canids (metacestodes and adult tapeworms), previously identified by PCR-RFLP, are a monophyletic group; (2) estimate how many evolutionary lineages are present within these samples; (3) assess whether tetrathyridial forms are members of a separate clade from the amorphous, acephalic metacestodes; and (4) evaluate the occurrence of asexual proliferation among selected isolates of Mesocestoides.

\section{MATERIALS AND METHODS}

\section{Specimen collection and morphological identification}

Metacestodes were recovered from fluid aspirated from the peritoneal cavity of naturally infected domestic dogs (Crosbie, Boyce, Platzer et al., 1998). Laboratory strains of tetrathyridia of $M$. corti were also obtained for analysis. Proglottids of Mesocestoides spp. were collected from the feces of coyotes (Canis latrans Say, 1823) and domestic dogs. Proglottids and tetrathyridia were identified as Mesocestoides spp. using the key of Rausch (1994). Acephalic metacestodes were identified using the molecular diagnostics described previously (Crosbie, Boyce, and Platzer et al., 1998). When DNA extraction could not be performed immediately, tapeworm samples were preserved in $70 \%$ ethanol.

\section{DNA extraction, PCR amplification, molecular identification, and sequencing}

DNA was extracted from cestode tissue as described previously (Crosbie, Boyce, and Platzer et al., 1998). Initial identification of all specimens was accomplished by evaluation of RFLP patterns for PCR amplicons from a 650-bp portion of the ITS 2 (Crosbie, Boyce, and Platzer et al., 1998). All PCR amplifications and sequencing reactions were performed in thin-walled microtubes in a Perkin Elmer GeneAmp 2400 thermocycler (Perkin Elmer Corporation, Foster City, California).

An 1,050-bp portion of DNA from the gene encoding 18S rRNA was amplified in 2 overlapping pieces using 2 pairs of primers for separate PCR reactions. The primer 18S-E (5'-CCGAATTCGTCGACAACCTGGTTGATCCTGCCAGT-3') that anneals at the $5^{\prime}$ end of the gene, adjacent to the external transcribed spacer (ETS); and primer A27 (5'-CCATACAAACGTCCCCGCCTG-3') that anneals near the center of the $18 \mathrm{~S}$ rDNA gene, amplified $\sim 700$ bp. The primers $18 \mathrm{~S}-8$ (5'-GCAGCCCGCGTAATTCCAGC-3') and Olson-6 (5'-ACGGAAACCTTGTTACGACT- $3^{\prime}$ ) also amplified $\sim 700 \mathrm{bp}$, extending from near the center of the $18 \mathrm{~S}$ rDNA gene toward the $3^{\prime}$ end. Total PCR volume for reactions with each pair of primers was $100 \mu \mathrm{l}$, comprised of $2 \mu \mathrm{l}$ of each primer $(10 \mu \mathrm{M}), 8 \mu \mathrm{l}$ of $10 \mathrm{mM}$ dNTP mix, $10 \mu \mathrm{l}$ of $10 \times$ PCR buffer with 1.5 $\mathrm{mM} \mathrm{MgCl} 2$ (final concentration), $0.2 \mu \mathrm{l}$ (5 units/ $\mu \mathrm{l}$ ) Taq Polymerase (Boehringer Mannheim, Indianapolis, Indiana), 1-5 $\mu$ l of template DNA (optimized to $\sim 250 \mathrm{ng}$ ), and a balance of sterile $\mathrm{H}_{2} \mathrm{O}$. For both sets of primers PCR conditions were: denaturation at $95 \mathrm{C}$ for $3 \mathrm{~min}$; then 2 cycles of 94 $\mathrm{C}$ for $30 \mathrm{sec}, 65 \mathrm{C}$ for $30 \mathrm{sec}$, and $72 \mathrm{C}$ for $30 \mathrm{sec}$ followed by 28 cycles of $94 \mathrm{C}$ for $30 \mathrm{sec}, 55 \mathrm{C}$ for $30 \mathrm{sec}$, and $72 \mathrm{C}$ for $30 \mathrm{sec}$, and final extension at $72 \mathrm{C}$ for $4 \mathrm{~min}$ before storage at $4 \mathrm{C}$. The presence and size of amplified DNA fragments was confirmed on $1.8 \%$ agarose gels containing $0.1 \mu \mathrm{g} /$ $\mathrm{ml}$ of ethidium bromide. Amplified DNA was purified using a QIAquick PCR Purification kit (Qiagen Inc., Chatsworth, California), and nucleic acid concentration determined by spectrophotometry.

Nucleotide sequencing was performed using fluorescent-labeled dideoxy cycle sequencing. Total PCR volume for each reaction was 20 $\mu \mathrm{l}$, comprised of $1 \mu \mathrm{l}$ of purified PCR template (optimized to $30 \mathrm{ng}$ ), $2 \mu \mathrm{l}$ of $10 \mu \mathrm{M}$ primer (18S-E, A27, 18S-8, or Olson-6), $9.5 \mu \mathrm{l}$ of terminator mix (Perkin Elmer), and $7.5 \mu \mathrm{l}$ of sterile $\mathrm{H}_{2} \mathrm{O}$. Cycle-sequencing conditions were: 25 cycles of $96 \mathrm{C}$ for $30 \mathrm{sec}, 50 \mathrm{C}$ for 15 $\mathrm{sec}$, and $60 \mathrm{C}$ for $4 \mathrm{~min}$ and final storage at $4 \mathrm{C}$. Reaction products were purified in Sephadex G-50 (Sigma, St. Louis, Missouri) columns and then vacuum dried. Each purified sample was rehydrated with $8 \mu 1$ of loading buffer (Perkin Elmer), and $2 \mu \mathrm{l}$ was electrophoresed using an ABI Prism 377 Sequencer (Perkin Elmer). Both strands were sequenced for all PCR products.

Amplification of ITS 2 fragments was accomplished as reported previously, using the primers NC-6 (5'-ATCGACATCTTGAACGCACATTGC-3') and NC-2 (5'-TTAGTTTCTTTTCCTCCGCT-3') (Gasser and Chilton, 1995; Crosbie, Boyce, and Platzer et al., 1998). Amplified DNA was purified and its concentration estimated by spectrophotometry. Each strand of the product was then sequenced as previously described, except that total PCR volume for each reaction was $20 \mu 1$, comprised of $10 \mu \mathrm{l}$ of purified PCR template (optimized to $\sim 30 \mathrm{ng}$ ), 2 $\mu \mathrm{l}$ of $10 \mu \mathrm{M}$ primer (either NC-6 or NC-2), and $8 \mu \mathrm{l}$ of terminator mix. Cycle-sequencing conditions were: 25 cycles of $96 \mathrm{C}$ for $10 \mathrm{sec}, 50 \mathrm{C}$ for $5 \mathrm{sec}$, and $60 \mathrm{C}$ for $4 \mathrm{~min}$ and final storage at $4 \mathrm{C}$ (Crosbie, Boyce, and Rodwell, 1998).

\section{Sequence alignment and phylogenetic analyses}

All sequence ambiguities for individual reactions were resolved by comparison of complementary strands, using the program Sequencher 3.0 (GeneCodes Corporation, Ann Arbor, Michigan). Initial multiple alignments of taxa were performed using ClustalW (Thompson et al., 1994). We first aligned the new 18 S rDNA data alone. This alignment was then aligned with the regions of $18 \mathrm{~S}$ rDNA sequence in common between our sequences and those of Mariaux (1998), using the profile alignment option in ClustalW. Neither of these alignments required any subsequent editing, due to the highly conserved 18S rDNA sequences. The ITS 2 sequences were aligned in ClustalW and then edited by eye (using MacClade 3.07; Maddison and Maddison, 1992) in order to increase aligned sequence similarity and improve inferences of positional homology. Aligned sequences were truncated so that terminal PCR primer sequences were not included in the analyses. Unambiguous contiguous gaps in the ITS 2 data set were recoded as a fifth character state (Crandall and Fitzpatrick, 1996).

Maximum parsimony (MP) and distance (neighbor-joining [NJ]) methods were used to estimate phylogenies, using PAUP*4.0d64. Heuristic searches were used for MP with tree-bisection reconnection branch swapping and random stepwise addition, with unrecoded gaps treated as missing data. Relative support for clades in MP and NJ analyses was assessed by bootstrap resampling using either 1,000 or 2,000 replicate data sets, as indicated. Different combinations of analyses were used for the 3 data sets. The alignment of the new 18S rDNA sequences was analyzed using midpoint rooted MP, and by NJ using log-determinant distances. For the profile alignment of $18 \mathrm{~S}$ rDNA sequences in common between our data and that of Mariaux (1998), similar analyses were performed, initially for all taxa. Subsequently we analyzed a lim- 
TABLE I. Specimen number, life history stage, host species, host location at time of collection, and date of collection of Mesocestoides spp.

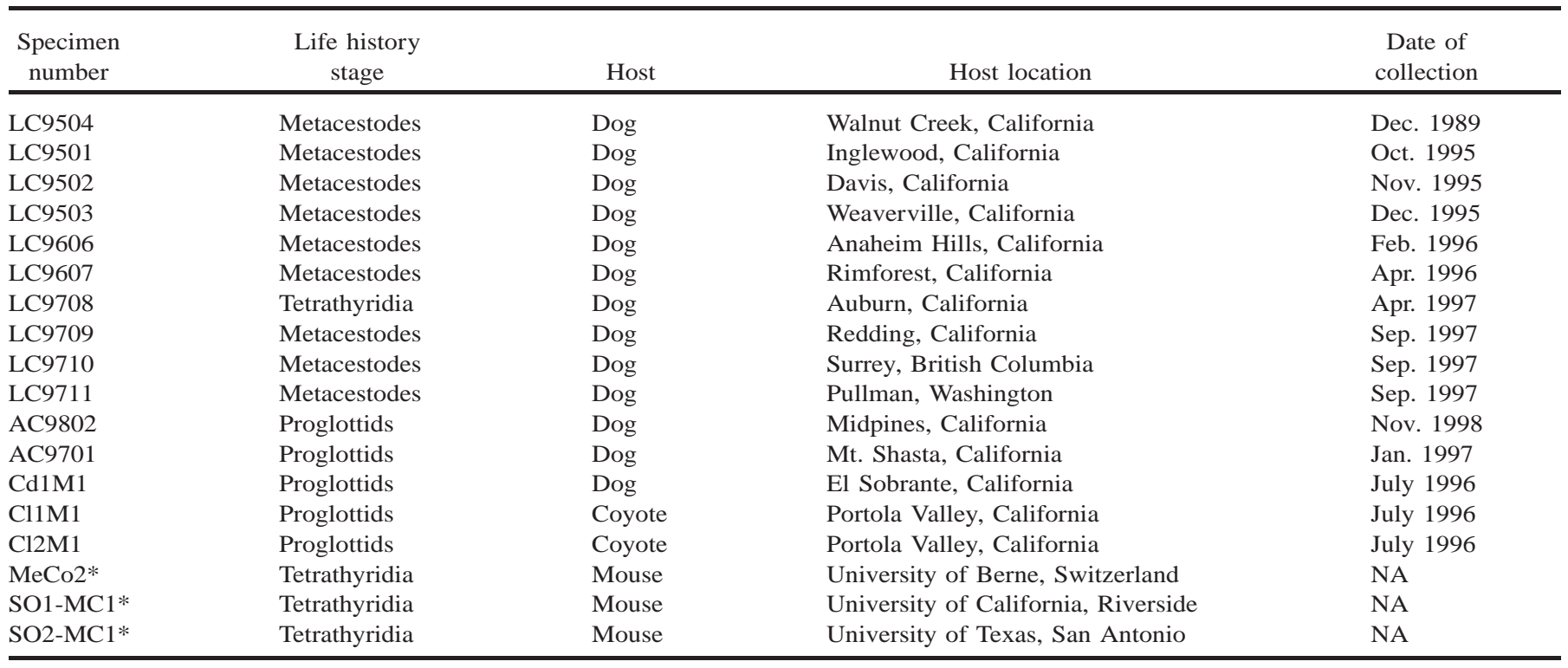

* Laboratory strains of M. corti, presumed original host a fence lizard (Specht and Voge, 1965).

ited data set of the Mesocestoides sequences and sequences from representatives of the 2 most closely related eucestode families, Nippotaeniidae (Amurotaenia Akhmerov, 1941), and Tetrabothriidae (Tetrabothrius), as inferred from the more inclusive analysis. The ITS 2 data set was also analyzed using MP and NJ, and trees were rooted based on the results of the $18 \mathrm{~S}$ rDNA analysis or were midpoint-rooted. The gaprecoded data set was only amenable to MP analysis.

\section{Asexual reproduction}

Samples of 3 isolates of acephalic metacestodes (LC9501, LC9502, LC9607) and tetrathyridia of isolate LC9708, each obtained from a different domestic dog (Table I), were inoculated into the peritoneal cavity of male BALB/cAnN mice (Simonsen Laboratories, Inc., Gilroy, California) that had been anesthetized with methoxyfluorane (Metofane, Pitman-Moore, Mundelein, Illinois). For each isolate, 30 metacestodes were inoculated into each mouse using a 16-gauge needle. Groups of 5 mice were inoculated with each isolate. Similarly, 30 metacestodes of laboratory isolates of $M$. corti (SO1-MC1, Table I) and T. crassiceps (both obtained from the University of California, Riverside) were also inoculated into groups of 5 mice. Mice were maintained on commercial rodent chow (Ralston Purina, St. Louis, Missouri) and water ad libitum. Ninety days postinoculation, all mice were euthanized by overinhalation of methoxyfluorane followed by retrorbital exsanguination and cervical dislocation. The abdominal and thoracic cavities of each mouse were opened and free metacestodes aspirated with a sterile Pasteur pipette. Metacestodes from each mouse were transferred to a petri dish containing phosphate-buffered saline and counted. The liver, spleen, heart, lungs, and diaphragm of each mouse were inspected for tissue invasion and were then preserved in $10 \%$ buffered formalin. Comparison of the numbers of metacestodes recovered for each isolate was conducted with Kruskal-Wallis ANOVA, with tapeworm isolate as the grouping variable. Where ANOVA showed significant differences, Mann-Whitney $U$-tests were used to compare specific isolates (Sokal and Rohlf, 1995). All statistical analyses were performed using SPSS (SPSS, 1993).

\section{RESULTS}

\section{Specimens}

We evaluated 18 samples of Mesocestoides in this study (Table I). Nine of these samples were composed of acephalic metacestodes recovered from peritoneal aspirates drawn from domestic dogs. An additional peritoneal sample (LC9708) from a dog contained identifiable tetrathyridia. These samples were obtained from dogs in California, Washington, and British Columbia, as described previously (Crosbie, Boyce, Platzer et al., 1998). Three samples of laboratory strains of $M$. corti tetrathyridia, assumed to be descendants of the original Specht and Voge (1965) material, were acquired from other institutions. The final 5 samples were proglottids recovered from the feces of dogs $(n=3)$ and coyotes $(n=2)$ in California.

\section{DNA extraction, PCR amplification, molecular identification, and sequencing}

Genomic DNA was successfully extracted from all 18 samples. Identification of each sample as Mesocestoides was accomplished by recognition of morphological characters (proglottids and tetrathyridia), and by the PCR-RFLP diagnostic technique (all samples) described previously (Crosbie, Boyce, and Platzer et al., 1998). A distinct RFLP pattern was observed from specimens AC9701 and AC9802, both proglottids recovered from dog feces (Table I). This RFLP pattern was different from previously examined samples of Mesocestoides (Table II) but was also different from the profiles of all other tapeworm genera and species previously analyzed (Crosbie, Boyce, Platzer et al., 1998). Representative material from each sample (where material remained after DNA extraction) was deposited at the United States National Parasite Collection, Beltsville, Maryland, USNPC 87366-372 and 88403-411.

The 18S rDNA and ITS 2 sequences were obtained for 14 and 18 , respectively, of the 18 Mesocestoides samples we collected. These sequences have been deposited in GenBank, accession numbers AF119677-AF119690 (18S rDNA sequences), and AF119691-AF119708 (ITS 2 sequences).

\section{Sequence alignment and phylogenetic analyses}

The final alignment of the 18S rDNA sequences of $14 \mathrm{Me}$ socestoides was $1,041 \mathrm{bp}$, of which 16 characters were variable 
TABLE II. Mesocestoides spp. restriction fragment length polymorphism (RFLP) patterns in a 650-bp fragment of the ITS 2 gene.

\begin{tabular}{lccccc}
\hline & Length of ITS 2 & & \multicolumn{2}{c}{ Restriction endonuclease, cut fragment sizes (bp) } \\
\cline { 3 - 6 } Specimen number & fragment (bp) & AluI & CfoI & Msp I & $210+400$ \\
\hline AC9701, AC9802 & 650 & 500 & 180 & $230+440$ & 575 \\
MeCo2, SO1-MC1* & 650 & 325 & 270 & $250+350$ & 575 \\
All other samples* & 650 & 380 & 270 & $250+350$ & \\
\hline
\end{tabular}

* Crosbie, Boyce, and Platzer et al. (1998).

and 3 parsimony-informative. These sequences were then aligned with the nucleotide alignment of Mariaux (1998), without any adjustments or recoding of gaps. Sixty-one eucestode taxa were included in this alignment that was $512 \mathrm{bp}$ in length (due to the limited overlap of the partial sequences of both studies), of which 221 characters were variable and 122 parsimony-informative. When only the 2 most closely related outgroups, Amurotaenia and Tetrabothrius (Mariaux, 1998), in the 61-taxon tree were considered with the 15 Mesocestoides se-

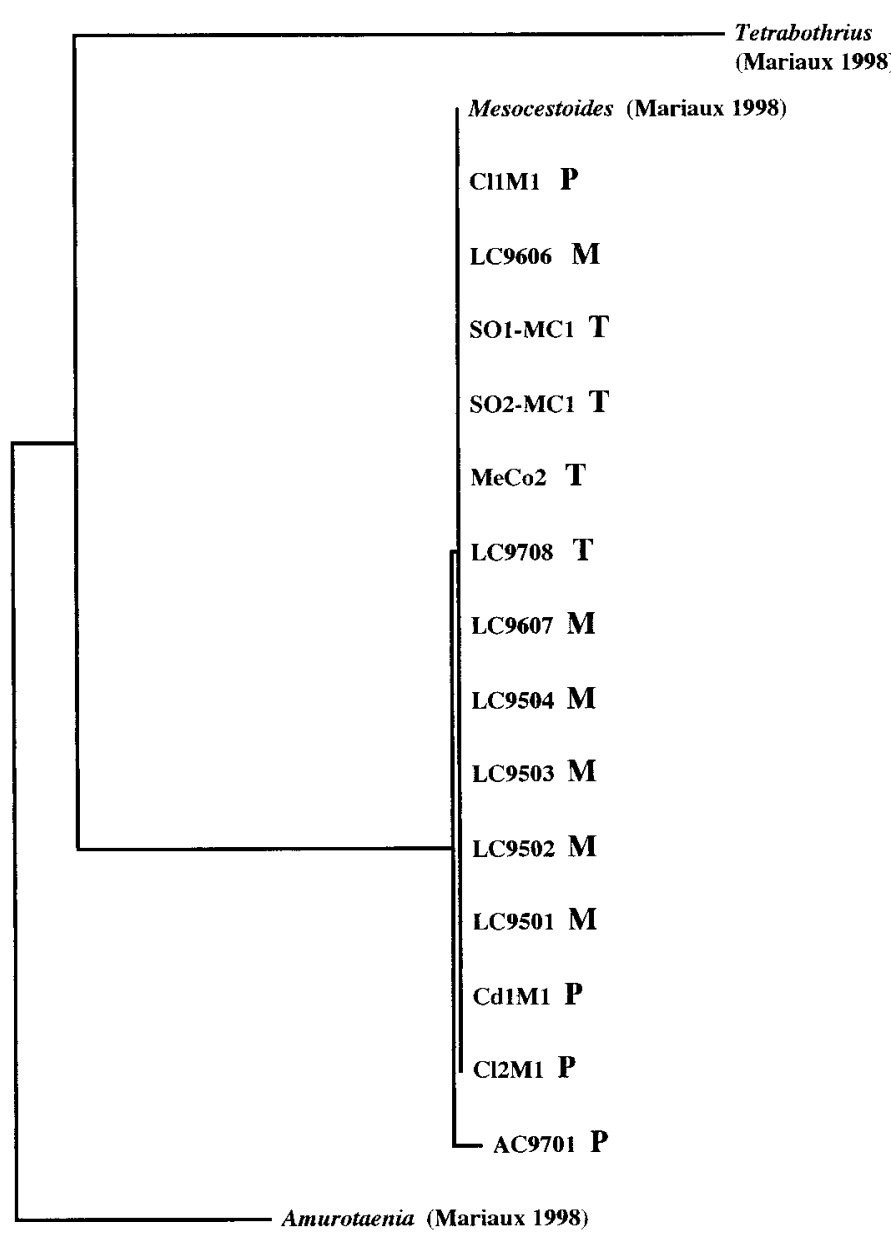

FIGURE 1. Phylogenetic relationship of 15 Mesocestoides spp. samples to Tetrabothrius sp. and Amurotaenia sp., derived from analysis of partial sequences of the nuclear 18S rDNA gene. Estimation of the phylogeny was by the neighbor-joining method based on a log-determinant distance matrix of 61 eucestode taxa. Branch lengths are proportional to genetic distance. Taxon label suffixes: $\mathrm{P}=$ proglottids, $\mathrm{M}$ $=$ metacestodes (acephalic), and $\mathrm{T}=$ tetrathyridia. Sample codes are as in Table I. quences (1 sequence from Mariaux [1998]), 41 of 512 characters were variable and 6 were parsimony-informative. These alignments and the ITS 2 alignment described below were deposited in TreeBASE (http://www.herbaria.harvard.edu/treebase/) (Sanderson et al., 1994).

Maximum parsimony analysis of the 61-taxon 18S rDNA data set (512 characters) and of the 17-taxon data set (512 characters) each yielded trees with an unresolved polytomy; the 61taxon analysis showed Mesocestoides as a monophyletic group. For both data sets, the Mesocestoides clade included all of our samples and the sequence obtained previously by Mariaux (1998) from a laboratory isolate of $M$. corti. NJ analysis of the rooted 61-taxon data set yielded a tree that showed the sequence from AC9701 (a proglottid from a dog) as basal to all other Mesocestoides, albeit with a short internal branch (Fig. 1). We subsequently rooted trees estimated from the ITS 2 data set with AC9701. The greater variability in the ITS 2 data precluded using non-Mesocestoides taxa as outgroups due to potential alignment uncertainties. The final unrecoded alignment of the ITS 2 sequences of 18 Mesocestoides was 499 bp in length, of which 216 characters were variable and 54 parsimony-informative. Recoding of unambiguous gaps yielded 22 additional characters, 7 of which were parsimony-informative.

Analyses of the ITS 2 data set by different methods resolved trees of broadly similar topology. The gap-recoded data set contained the most parsimony-informative characters, and bootstrapped (Fig. 2) and consensus (Fig. 3) trees generated from MP analysis are shown. MP analysis with midpoint rooting resolved a clade composed of AC9701 and AC9802 as basal to the other Mesocestoides, which was consistent with the position of AC9701 in the 18S rDNA analysis (Fig. 1). AC9802 represents proglottids recovered recently from a domestic dog (Table I) subsequent to completion of the $18 \mathrm{~S}$ rDNA analysis. This specimen was very similar to AC9701, differing at only 2 of 521 ITS 2 sites, and it had the same RFLP profile (Table II). Aside from this basal group, 2 other clades were strongly supported by MP bootstrap analysis (Fig. 2). One clade (100\% support) was composed of the 3 isolates of $M$. corti and the single isolate of tetrathyridia from a dog (LC9708). Within this clade, moderate support $(69 \%)$ was found for a clade composed of the $3 \mathrm{M}$. corti isolates, each of which had identical ITS 2 sequences. The second strongly supported (82\%) clade was composed of all of the remaining specimens of Mesocestoides spp. that included all of the acephalic metacestodes and proglottids from coyotes and 1 domestic dog. Within this clade, there was weak to moderate support $(54,66$, and $66 \%)$ for 3 subclades (Fig. 2).

A strict consensus MP tree (Fig. 3) based on the ITS 2 sequences revealed 6 distinct apomorphy-defined terminal line- 


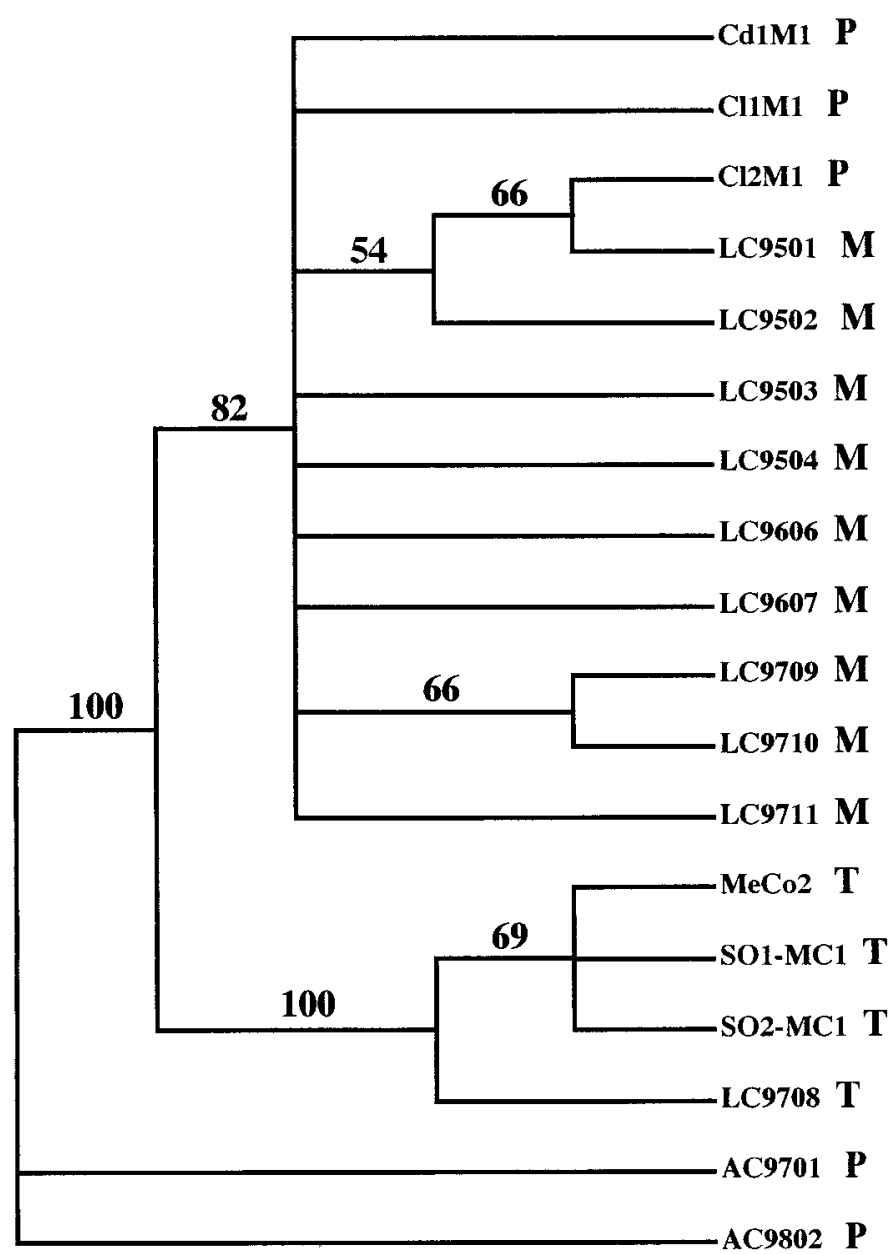

FIGURE 2. Estimated phylogeny of 18 Mesocestoides spp. samples based on partial sequences of the ITS 2 gene, analyzed by maximum parsimony after recoding of unambiguous gaps in the alignment. Numbers above branches represent bootstrap support where greater than $50 \%$ (2,000 replicates). Taxon label suffixes: $\mathrm{P}=$ proglottids, $\mathrm{M}=$ metacestodes (acephalic), and $\mathrm{T}=$ tetrathyridia. Sample codes are as in Table I.

ages. The outgroup lineage represented by AC9701 and AC9802 was only recovered through distance analyses of the $18 \mathrm{~S}$ rDNA data or by midpoint-rooted analysis of the ITS 2 data. The strict consensus MP analysis also recovered the major clades that were identified in the bootstrap analysis (Fig. 2) that are also labeled in Figure 3.

\section{Asexual reproduction}

Three of the 4 isolates from dogs reproduced asexually in the peritoneal cavity of mice, as did the isolates of $M$. corti and T. crassiceps (Table III). Isolate LC9502 did not show evidence of asexual reproduction. Kruskal-Wallis ANOVA showed a significant difference between isolates in numbers of metacestodes recovered $(H=15.448$, df $=5, P=0.009)$. Significantly larger numbers of tetrathyridia of isolate LC9708 were recovered compared with isolates LC9501, LC9502, LC9607, and T. crassiceps $(U=0.000, P=0.014$ in each case). There was no significant difference between the numbers of tetrathyridia recovered of LC9708 compared with those of $M$. corti $(U=$ 2.000, $P=0.083$ ). The 3 acephalic isolates (LC9501, LC9502,

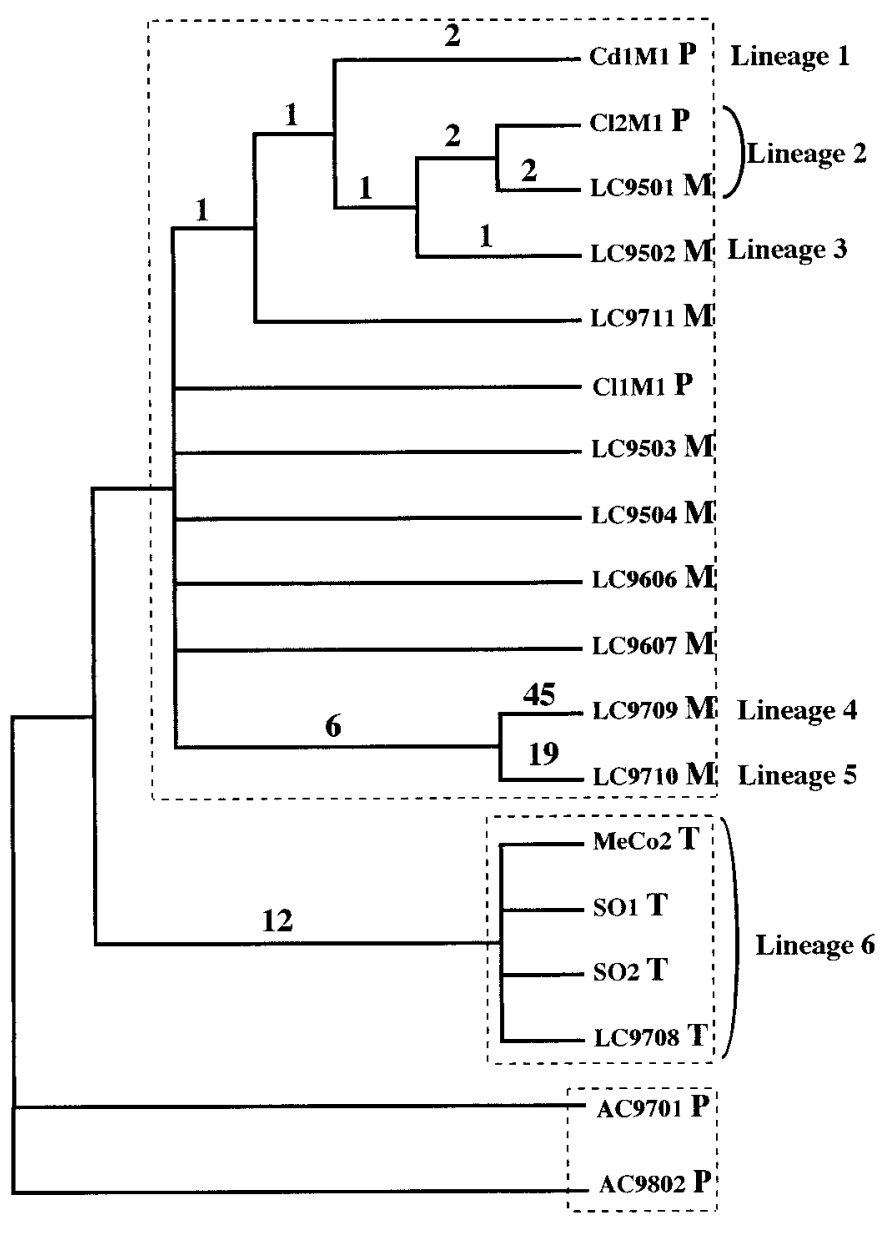

FIGURE 3. Maximum parsimony (strict consensus of 302 trees, tree length $=309$, consistency index $=0.71$, homoplasy index $=0.29$, retention index $=0.81$ ) estimated phylogeny of 18 Mesocestoides spp. samples based on partial sequences of the ITS 2 gene, after recoding of unambiguous gaps in the alignment. Numbers above branches represent the numbers of unambiguous apomorphies (those present in all reconstructions). Taxon label suffixes: $\mathrm{P}=$ proglottids, $\mathrm{M}=$ metacestodes (acephalic), and $\mathrm{T}=$ tetrathyridia. Sample codes are as in Table I. Three potential topology-based (combined distance and parsimony analysis) lineages are indicated by boxes surrounding clades, 6 apomorphy-defined lineages by notation to the right of terminal tree branches.

and LC9607) and T. crassiceps did not invade tissues. In contrast, invasion of the liver by tetrathyridia of LC9708 was seen in each mouse, with extensive areas of calcification. Mesocestoides corti tetrathyridia were found in the livers of 3 of 4 mice, and numerous tetrathyridia were recovered from the scrotal sac in the same 3 mice.

\section{DISCUSSION}

This study represents an extensive analysis of sequence variation within Mesocestoides infecting canids in western North America, the first assessment of intrageneric evolutionary relationships within the Mesocestoididae, and the first evidence of asexual reproduction in Mesocestoides isolates other than laboratory isolates of $M$. corti. We interpret our results (specifically our analyses of the $18 \mathrm{~S}$ rDNA data) as clearly indicating that all of the specimens we analyzed form a monophyletic 
TABLE III. Metacestodes recovered from mice 90 days after peritoneal inoculation with 30 metacestodes.

\begin{tabular}{|c|c|c|c|c|c|}
\hline LC9501 & 5 & 62 & 82.0 & 23 & $3-200$ \\
\hline LC9607 & 5 & 62 & 40.3 & 63 & $15-122$ \\
\hline LC9708 & 4 & 1,154 & 496.8 & 1,121 & $704-1,670$ \\
\hline M. corti & 4 & 567 & 493.6 & 501 & $55-1,211$ \\
\hline
\end{tabular}

* One mouse in each of the LC9708 and M. corti groups died at $<90$ days.

group and are members of Mesocestoides. Thus, the 18S rDNA data proved particularly valuable for identifying the amorphous, acephalic metacestodes from dogs in a phylogenetic context.

The segments of the $18 \mathrm{~S}$ rDNA gene we sequenced only partially overlapped the region sequenced by Mariaux (1998), resulting in a common alignment of 512 nucleotides. Given the difference in the number of characters (1,102 in Mariaux [1998] vs. 512 in this study) between the alignments, it is not unexpected that the result of the MP search yielded different results than those reported by Mariaux (1998). Specifically, our analysis showed Tetrabothrius as the basal sister taxon of Mesocestoides, with Amurotaenia basal to both (Fig. 1), whereas Mariaux's analysis showed the nippotaeniid Amurotaenia basal to Mesocestoides that was in turn basal to Tetrabothrius, which was then basal to all other Cyclophyllidea (Mariaux, 1998 [Fig. 2]). Our results presented here are congruent with all morphologically based phylogenetic analyses (Hoberg et al., 1997; Hoberg, Gardner, and Campbell, 1999; Hoberg, Jones, and Bray, 1999).

We did detect considerable variation among ITS 2 sequences of these isolates. We interpret our analysis of the ITS 2 data, coupled with the analysis of the $18 \mathrm{~S}$ rDNA data, to indicate the existence of several distinct evolutionary lineages within $\mathrm{Me}$ socestoides parasitizing canids in western North America. Conservatively, we identify a minimum of 3 distinct lineages, as indicated by the boxes drawn within Figure 3. One lineage is represented by AC9701 and AC9802, both proglottids from dogs. This lineage was identified based on distance analysis of the $18 \mathrm{~S}$ rDNA sequences. A second lineage, well supported by bootstrap analysis (100\%), is composed of the $3 \mathrm{M}$. corti isolates (that we assume to be derived from the original Specht and Voge [1965] isolate) and the single isolate of tetrathyridia (LC9708) we obtained from a dog in California (Table I). The morphological similarity among the tetrathyridia is notable, and it is possible that these samples represent the same species, either $M$. corti or the proposed M. vogae (Etges, 1991). Significantly, both $M$. corti and LC9708 invaded tissues during asexual reproduction, potentially indicating a biological difference between members of this lineage and the amorphous, acephalic metacestodes, 3 isolates of which did not invade tissues in the present study. Notably, tissues were not invaded by the acephalic metacestodes of the $T$. crassiceps isolate used in the present study. The third lineage is represented by all of the remaining sequences analyzed and includes the acephalic metacestodes from dogs and proglottids from a dog and 2 coyotes (Table I, Fig. 3). Bootstrap support for this group is also moderately strong $(82 \%)$.
Adams (1998) has proposed adopting a phylogenetic approach to designation of species in nematology in order to avoid predictive systematic errors inherent in phenetic and biological species concepts. Applying this methodology to the ITS 2 data unambiguously identifies 6 lineages based on apomorphies (Fig. 3 ). Of these 6 lineages, 1 is the $M$. corti/M. vogae group, found naturally as tetrathyridia in fence lizards (Specht and Voge, 1965) and found as tetrathyridia (LC9708) in a dog in the present study. Three other lineages have been sampled only as acephalic metacestodes in dogs (lineages 3-5), 1 as metacestodes in dogs and proglottids in coyotes (lineage 2), and 1 as proglottids in coyotes (lineage 1). Asexual proliferation was seen within lineage 2 as represented by LC9501 (Table III). Lineage 3 (LC9502) did not show asexual reproduction in the present study. One other isolate (LC9607) replicated asexually (Table III); this isolate is not a member of an unambiguous lineage. The prevalence of asexual proliferation in Mesocestoides is still unknown, but it is evident that it occurs in more than 1 evolutionary lineage as identified here, and that invasion of tissues by metacestodes is not a universal characteristic. In the present study, only tetrathyridia proved capable of tissue invasion in laboratory mice. Whether acephalic isolates are capable of tissue invasion in dogs is unknown.

Without further morphological and ecological evidence we cannot assess if the lineages we identified are congruent with distinct species. However, the ITS 2 data should be useful for matching adult cestodes with the metacestodes characterized in this study, and this marker may be useful for tracking life-cycle stages in field or experimental settings.

Current estimations of eucestode phylogeny are supported at the familial level by both morphological and genetic data, as shown by the collective work of Hoberg et al. (1997), Hoberg, Gardner, and Campbell (1999), Hoberg, Jones, and Bray (1999), and Mariaux (1998). In recent years, 18S rDNA sequences have been used extensively for phylogenetic inference at the suprageneric level, including within the Platyhelminthes and within the Eucestoda (Mariaux, 1998). Justine (1998) has added support to the evolving paradigm of cestode phylogeny by analysis of spermatozoon characteristics within the Eucestoda. Recently, spermatozoon ultrastructure and spermiogenesis in $M$. literratus were shown to be different from all other cyclophyllideans (Miquel et al., 1999), which provides further support for exclusion of the Mesocestoididae from the Cyclophyllidea, as previously suggested (Wardle and McLeod, 1952; Brooks et al., 1991). Other morphological characters that may prove useful were reviewed by Mariaux (1996).

The ITS 2 data provide a framework for comparison of ad- 
ditional metacestode and adult tapeworms from dogs and those from other wild vertebrates. Sampling additional hosts from single geographic locations would be important in assessing the distribution of evolutionary lineages and for applying apomorphy-based phylogenetic species definitions (Adams, 1998).

Although we have presented additional genetic evidence regarding the Mesocestoididae and have demonstrated the occurrence of asexual reproduction in isolates other than $M$. corti, species distinctions and life cycles remain uncertain (Etges, 1991). Additional studies of mesocestoidid life cycles are critically required. The equivocal evidence of the existence of an arthropod as the first intermediate host (Soldatowa, 1944) demands further investigation and can now be aided by analysis of diagnostic genetic markers. Additionally, the role of wild vertebrates (particularly coyotes and fence lizards) in the population dynamics of Mesocestoides in western North America needs to be investigated, not the least for the implications for companion animal health. Detailed knowledge of the life cycles of Mesocestoides spp. is needed (Crosbie, Boyce, Platzer et al., 1998 ) in order to provide the information necessary to advise owners of domestic animals about transmission risks.

\section{ACKNOWLEDGMENTS}

G. Lee is acknowledged for her technical assistance. P. D. Olson of the Department of Zoology, The Natural History Museum, London is thanked for providing 2 of the primer sequences used for the 18S rDNA amplifications. D. Swofford (Smithsonian Institution) kindly allowed us to publish analyses conducted using PAUP*4.0d64. E. P. Hoberg and R. Lichtenfels are thanked for their prompt accession of samples and assistance with morphological identification. We also acknowledge the cooperation of the veterinarians who provided us with the clinical samples from dogs. This project was partially supported by a grant from the Center for Companion Animal Health, School of Veterinary Medicine, University of California, Davis.

\section{LITERATURE CITED}

ADAMS, B. J. 1998. Species concepts and the evolutionary paradigm in modern nematology. Journal of Nematology 30: 1-21.

Adlard, R. D., S. C. Barker, D. Blair, and T. H. Cribb. 1993. Comparison of the second internal transcribed spacer (ribosomal DNA) from populations and species of Fasciolidae (Digenea). International Journal for Parasitology 23: 423-425.

Barker, S. C., D. Blair, T. H. Cribb, and K. Tonion. 1993. Phylogenetic position of Heronimus mollis (Digenea): Evidence from $18 \mathrm{~S}$ ribosomal RNA. International Journal for Parasitology 23: 533-536.

Baverstock, P. R., R. Fielke, A. M. Johnson, R. A. Bray, and I. BEVERIDGE. 1991. Conflicting phylogenetic hypotheses for the parasitic platyhelminths tested by partial sequencing of $18 \mathrm{~S}$ ribosomal RNA. International Journal for Parasitology 21: 329-339.

BLAIR, D. 1993. The phylogenetic position of the Aspidobothrea within the parasitic flatworms inferred from ribosomal RNA sequence data. International Journal for Parasitology 23: 169-178.

—, AND S. C. BARKER. 1993. Affinities of the Gyliauchenidae: Utility of the 18S rRNA gene for phylogenetic inference in the Digenea (Platyhelminthes). International Journal for Parasitology 23: $527-532$.

Brooks, D. R., E. P. Hoberg, AND P. J. Weekes. 1991. Preliminary phylogenetic systematic analysis of the major lineages of the Eucestoda (Platyhelminthes: Cercomeria). Proceedings of the Biological Society of Washington 104: 651-668.

Campos, A., M. P. Cummings, J. L. Reyes, and J. P. Laclette. 1998. Phylogenetic relationships of Platyhelminthes based on $18 \mathrm{~S}$ ribo- somal gene sequences. Molecular Phylogenetics and Evolution 10: $1-10$.

Carranza, S., J. Baguña, and M. Riutort. 1997. Are the Platyhelminthes a monophyletic primitive group? An assessment using 18S rDNA sequences. Molecular Biology and Evolution 14: 485-497.

- I. Ruiz-Trillo, D. T. J. Littlewood, M. Ruitort, AND J. BAGUÑA. 1998. A reappraisal of the phylogenetic and taxonomic position of land planarians (Platyhelminthes, Turbellaria, Tricladida) inferred from 18S rDNA sequences. Pedobiologia 42: 433-440.

ConN, D. B. 1990. The rarity of asexual reproduction among Mesocestoides tetrathyridia (Cestoda). Journal of Parasitology 76: 453-455.

Crandall, K. A., AND J. F. FitzPatrick, JR. 1996. Crayfish molecular systematics: Using a combination of procedures to estimate phylogeny. Systematic Biology 45: 1-26.

Crosbie, P. R., W. M. Boyce, E. G. Platzer, S. A. Nadler, and C. KERNER. 1998. Diagnostic procedures and treatment of eleven dogs with peritoneal infections caused by metacestodes of Mesocestoides spp. Journal of the American Veterinary Medical Association 213: $1578-1583$.

- - AND T. C. RoDwell. 1998. DNA sequence variation in Dermacentor hunteri and estimated phylogenies of Dermacentor spp. (Acari: Ixodidae) in the New World. Journal of Medical Entomology 35: 277-288.

Etges, F. R. 1991. The proliferative tetrathyridium of Mesocestoides vogae sp. $\mathrm{n}$. (Cestoda). Journal of the Helminthological Society of Washington 58: 181-185.

Gasser, R. B., AND N. B. Chilton. 1995. Characterization of taeniid cestode species by PCR-RFLP of ITS-2 ribosomal DNA. Acta Tropica 59: 31-40.

Hoberg, E. P., S. L. Gardner, And R. A. CAmpbell. 1999. Systematics of the Eucestoda: Advances towards a new phylogenetic paradigm, and observations on the early diversification of tapeworms and vertebrates. Systematic Parasitology 42: 1-12.

, A. JONES, AND R. A. BRAY. 1999. Phylogenetic analysis among the families of the Cyclophyllidea (Eucestoda) based on comparative morphology, with new hypotheses for co-evolution in vertebrates. Systematic Parasitology 42: 51-73.

, J. Mariaux, J.-L. Justine, D. R. Brooks, and P. J. Weekes. 1997. Phylogeny of the orders of the Eucestoda (Cercomeromorphae) based on comparative morphology: Historical perspectives and a new working hypothesis. Journal of Parasitology 83: 11281147.

HoEpPLI, R. J. C. 1925. Mesocestoides corti, a new species of cestode from the mouse. Journal of Parasitology 12: 91-96.

Justine, J.-L. 1998. Spermatozoa as phylogenetic characters for the Eucestoda. Journal of Parasitology 84: 385-408.

Katayama, T., M. Nishioka, And M. Yamamoto. 1996. Phylogenetic relationships among turbellarian orders inferred from 18S rDNA sequences. Zoological Science 13: 747-756.

- M. Yamamoto, H. Wada, and N. Satoh. 1993. Phylogenetic position of acoel turbellarians inferred from partial 18S rDNA sequences. Zoological Science 10: 529-536.

Lumb, S. M., R. A. Bray, AND D. Rollinson. 1993. Partial small (18S) rRNA gene sequences from fish parasites of the families Lepocreadiidae and Fellodistomidae (Digenea) and their use in phylogenetic analyses. Systematic Parasitology 26: 141-149.

MadDison, W. P., AND D. R. Maddison. 1992. MacClade: Analysis of phylogeny and character evolution, version 3.07. Sinauer Associates, Sunderland, Massachusetts.

MariauX, J. 1996. Cestode systematics: Any progress? International Journal of Parasitology 26: 231-243.

- 1998. A molecular phylogeny of the Eucestoda. Journal of Parasitology 84: 114-124.

Miquel, J., C. Feliu, and B. Marchand. 1999. Ultrastructure of spermiogenesis and the spermatozoon of Mesocestoides litteratus (Cestoda, Mesocestoididae). International Journal for Parasitology 29: 499-510.

Rausch, R. L. 1994. Family Mesocestoididae Fuhrmann, 1907. In Keys to the cestode parasites of vertebrates, L. F. Khalil, A. Jones, and R. A. Bray (eds.). CAB International, Wallingford, U.K., p. 309314.

Rohde, K., C. Hefford, J. T. Ellis, P. R. Baverstock, A. M. Johnson, N. A. Watson, and S. Dittman. 1993. Contributions to the phy- 
logeny of Platyhelminthes based on partial sequencing of $18 \mathrm{~S}$ ribosomal DNA. International Journal for Parasitology 23: 705-724.

, K. Luton, P. R. Baverstock, And A. M. Johnson. 1994. The phylogenetic relationships of Kronborgia (Platyhelminthes, Fecampiida) based on comparison of $18 \mathrm{~S}$ ribosomal DNA sequences. International Journal for Parasitology 24: 657-669.

Sanderson, M. J., M. J. Donoghue, W. Piel, And T. Eriksson. 1994. TreeBASE: A prototype database of phylogenetic analyses and an interactive tool for browsing the phylogeny of life. American Journal of Botany 81: 183 .

SoKaL, R. R., AND F. J. Rohlf. 1995. Biometry: The principles and practice of statistics in biological research, 3rd ed. Freeman, New York, New York.

SoldatowA, A. P. 1944. A contribution to the study of the development cycle in the cestode Mesocestoides lineatus (Goeze 1782), parasite of carnivorous mammals. Doklau Akademia Nauk SSSR 45: 310_ 312.

Specht, D., AND M. Voge. 1965. Asexual multiplication of Mesocestoides tetrathyridia in laboratory animals. Journal of Parasitology 52: $268-272$.

SPSS FOR WINDOws. 1993. Base system user's guide, release 6.0. SPSS, Chicago, Illinois.

Thompson, J. D., D. G. Higgins, And T. J. Gibson. 1994. ClustalWImproving the sensitivity of progressive multiple sequence alignment through sequence weighting, position-specific gap penalties and weight matrix choice. Nucleic Acids Research 22: 4673-4680.

Wardle, R. A., AND J. A. MCLeOD. 1952. The zoology of tapeworms. University of Minnesota Press, Minneapolis, Minnesota, $780 \mathrm{p}$. , - - AND S. RADINOVSKY. 1974. Advances in the zoology of tapeworms 1950-1970. University of Minnesota Press, Minneapolis, Minnesota, $274 \mathrm{p}$. 